\title{
DISTRIBUTION NETWORK RECONFIGURATION FOR POWER LOSS MINIMIZATION AND VOLTAGE PROFILE ENHANCEMENT USING ANT LION ALGORITHM
}

\author{
Maryam SHOKOUHI ${ }^{1}$, Shahrokh SHOJAEIAN ${ }^{2}$ \\ ${ }^{1,2}$ Department of Engineering, Islamic Azad University, Khomeinishahr Branch \\ P,O,Box 84175-119, Khomainishahr, Isfahan, IRAN \\ ${ }^{1}$ maryam.shokouhi@iaukhsh.ac.ir \\ 2shojaeian@iaukhsh.ac.ir
}

\begin{abstract}
Distribution networks are designed as a ring and operated as a radial form. Therefore, the reconfiguration is a simple and cost-effective way to use existing facilities without the need for any new equipment in distribution networks to achieve various objectives such as: power loss reduction, feeder overload reduction, load balancing, voltage profile improvement, reducing the number of switching considering constraints that ultimately result in the power loss reduction. In this paper, a new method based on the Ant Lion algorithm (a modern meta-heuristic algorithm) is provided for the reconfiguration of distribution networks. Considering the extension of the distribution networks and complexity of their communications networks, and the various parameters, using smart techniques is inevitable. The proposed approach is tested on the IEEE 33 \& 69-bus radial standard distribution networks. The Evaluation of results in MATLAB software shows the effectiveness of the Ant Lion algorithm in the distribution network reconfiguration.
\end{abstract}

Keywords:Ant Lion (AL) algorithm, power loss, reconfiguration, voltage profile

\section{Introduction}

Energy distribution network is the last link between generation and consumption, thus it seems that this sector is very important. So many ways has been created to improve the efficiency of the distribution network. One of the most basic and most common ways to improve the performance of the distribution network is the network reconfiguration. The distribution network voltage is low and as a result high current and therefore distribution power loss is far more important than the transmission network. Energy loss of the power grids causes significant waste quantities of electrical energy and thus imposes huge sums to power companies. The cost of losses includes investment costs for new facilities, blackout and environmental damages caused by generation as well as decrease in income. In the meantime, the major share of power grid losses is related to distribution networks. The task of distribution networks is electric power transmission from sub-transmission substations or small-scale power plants to consumers and the voltage level change depended on the case and based on the necessities. In distribution networks, the ring branching is considered for feeder changes, in order to reduce losses and improve voltage profile, but during power supplying the network configuration should be in such a way that there is no ring. There are several techniques for reducing losses in distribution networks. The most common methods can be referred to the capacitor placement, changing the system feeders and find the optimum source location and reconfiguration. Network reconfiguration is the easiest and least expensive method to reduce losses. In this way, the power path from the source to the load varies while loss is minimized as well as the radial network is maintained. In the distribution network feeders, there are two switch types, normally close and normally open. Open or close status of the switches is done by the distribution network reconfiguration, to achieve a new configuration in order to meet the desired objectives such as reducing power losses, load balancing, improving the voltage profile, improving the power quality, service to all consumers, minimizing areas without electricity and increasing the network reliability.

In the literature, several ways are proposed to reconfigure for different purposes. Reconfiguration problem is known as a combinational nonlinear 
optimization problem with multiple constraints. Some configurations are not allowed to choose because of the violation of constraints such as radial network, the energizing at all nodes and the violation of operation constraints such as allowable limits for voltage and current.

First the reconfiguration problem was done by Back and Merlin in 1791 to reduce active power loss with an innovative approach. In this method, to achieve optimal configuration, after closing all the switches, it is started to open switches one by one of which current is lower [1]. Then, various techniques were provided to reconfigure distribution networks, such as the innovative method introduced by $\mathrm{Wu}$ and Baran. In this method, also all switches are considered to be closed. Then by a power flow, a ring which has the most of loss is selected and in that ring a branch where there is the most of loss is selected and opened. The process will continue to open the last switch of the last ring as long as the best configuration is achieved [2]. In addition to innovative techniques, smart algorithms are used to find the optimal configuration such as genetic algorithms, differential evolution algorithm, bacteria foraging algorithm, ant colony algorithm and particle swarm algorithm and neural network [3-7].

All these algorithms based on the inspiration of a phenomenon in nature, are able to achieve a mathematical model that can be used to optimize issues such as the reconfiguration of distribution network. A variety of purposes including active power loss reduction, voltage profile improvement, load balancing improvement and improvement of reliability indexes are considered as the objective function in the optimization algorithms.

In general, in the network reconfiguration, focus is more on reducing loss. Since, the distribution network reconfiguration is a multi-objective problem; the simultaneous optimization of two or more goals could bring better results.

In [8] a new multi-objective method combining genetic algorithm and fuzzy logic is used to solve the of distribution network reconfiguration problem. In this research, five goals in mind are converted to the fuzzy framework and the reconfiguration problem through the obtained objective function is solved by a genetic adaptive algorithm.

Also, Mendoza and at al. were able to use the micro genetic multi-objective algorithm in order to simultaneously optimize the power loss at several stages together with one of the reliability indicators in which points were obtained in each stage known as the Pareto Front [9].

In [10], load balancing and loss reduction are addressed by using the multi-objective genetic algorithm with not dominated sorting version 2 (NSGA II).

Also, in [2] load balancing and loss reduction are addressed has been multi-objective particle swarm optimization (PSO) algorithm. Simulation results show that the algorithm is better than multi-objective genetic algorithm to find the Pareto points.

In this paper, to correctly understand the effect of reconfiguration on the power loss and voltage profile index in distribution systems, 33 \& 69-busIEEE standard systems are simulated in MATLAB $^{\circledR}$ software. The reconfiguration problem to reduce the power loss and improve the network voltage profile is done by Ant Lion algorithm taking into account limitations such as maximum and minimum voltage deviation, limited line power transmission capacity and system operation with a radially topology.

\section{Problem Formulation}

For reconfiguration problem, the radial nature of the network is considered as a very important constraint. The objective function (O.F.) of the problem is formulated to minimize the power loss and voltage deviation of the distribution networks and is given as

$$
\begin{aligned}
& F_{2}=\sum_{i=1}^{n B \text { Bus }}\left|V_{\text {rated }}-V_{i}\right| \\
& F_{1}=\sum_{j=1}^{n \text { Line }} P_{\text {LOSS }, \mathrm{j}} \\
& \text { O.F. }=w_{1} \times \sum_{i=1}^{n \text { Bus }}\left|V_{\text {rated }}-V_{i}\right|+w_{2} \times \sum_{j=1}^{n L \text { Line }} P_{\text {LOSS }, j}
\end{aligned}
$$

The first part is related to the voltage profile improvement and the second part is related to power loss reduction.

Subject to the following operational constraints:

1) Voltage limit:

$V_{i, \text { min }} \leq V_{i} \leq V_{i, \max }$

2) Reactive power compensation limit:

$Q_{c i, \min } \leq Q_{c i} \leq Q_{c i \text { max }}$

3) Line power capacity limit:

$S_{l i} \leq S_{l i \text { max }}$

Furthermore, the radial nature of distribution network must be maintained, and all loads must be served. If any one of the above constraints is violated, then the resultant solution will be rejected.

\section{Optimization Algorithm}

Ant Lions (doodlebugs) belong to the Myrmeleontidae family and Neuroptera order (netwinged insects). The lifecycle of Ant Lions includes two main phases: larvae and adult. Their names originate from their unique hunting behavior and their favorite prey. An Ant Lion larvae digs a cone-shaped pit in sand by moving along a circular path and throwing out sands with its massive jaw. Another interesting behavior that has been observed in life style of Ant Lions is the relevancy of the size of the trap and two things: level of hunger and shape of the moon. Ant Lions tend to dig out larger traps as they become hungrier and/or when the moon is full [11].

\section{A) Operators of the AL algorithm}

The AL algorithm mimics interaction between Ant Lions and ants in the trap. To model such 
interactions, ants are required to move over the search space, and Ant Lions are allowed to hunt them and become fitter using traps. Since ants move stochastically in nature when searching for food, a random walk is chosen for modeling ants' movement as follows:

$x(t)=\left[0\right.$, cumsum $\left(2 r\left(t_{1}\right)-1\right)$, cumsum $\left(2 r\left(t_{2}\right)-1\right), \ldots$, cumsum(2r(t $\left.\left.\left.t_{n}\right)-1\right)\right]$

$r(t)$ is a stochastic function defined as follows:

$r(t)= \begin{cases}1 & \text { if rand }>0.5 \\ 0 & \text { if rand } \leq 0.5\end{cases}$

\section{A.1) Operators of the AL algorithm}

In order to keep the random walks inside the search space, they are normalized using the following equation (min-max normalization):

$$
X_{i}^{t}=\frac{\left(X_{i}^{t}-a_{i}\right) \times\left(d_{i}-c_{i}^{t}\right)}{d_{i}^{t}-a_{i}}+c_{i}
$$

\section{A.2) Sliding ants towards Ant Lion}

With the mechanisms proposed so far, Ant Lions are able to build traps proportional to their fitness and ants are required to move randomly. However, Ant Lions shoot sands outwards the center of the pit once they realize that an ant is in the trap. This behavior slides down the trapped ant that is trying to escape. For mathematically modeling this behavior, the radius of ants's random walks hyper-sphere is decreased adaptively. The following equations are proposed in this regard:

$$
\begin{aligned}
& c^{t}=\frac{c^{t}}{I} \\
& d^{t}=\frac{d^{t}}{I}
\end{aligned}
$$

\section{A.3) Catching prey and re-building the pit}

The final stage of hunt is when an ant reaches the bottom of the pit and is caught in the Ant Lion's jaw. After this stage, the Ant Lion pulls the ant inside the sand and consumes its body. For mimicking this process, it is assumed that catching prey occur when ants becomes fitter (goes inside sand) than its corresponding Ant Lion. An Ant Lions then required to update its position to the latest position of the hunted ant to enhance its chance of catching new prey. The following equation is proposed in this regard:

$$
\text { Antlion }_{j}^{t}=\text { Ant }_{i}^{t} \quad \text { if } \quad f\left(\text { Ant }_{i}^{t}\right)>f\left(\text { Antlion }_{j}^{t}\right)
$$

A.5) Elitism

In this study the best Ant Lion obtained so far in each iteration is saved and considered as an elite. Therefore, it is assumed that every ant randomly walks around a selected Ant Lion by the roulette wheel and the elite simultaneously as follows:

$$
A n t_{i}^{t}=\frac{R_{A}^{t}+R_{E}^{t}}{2}
$$

\section{B) AL algorithm}

The pseudo codes of the AL algorithm are defined as follows [11]:

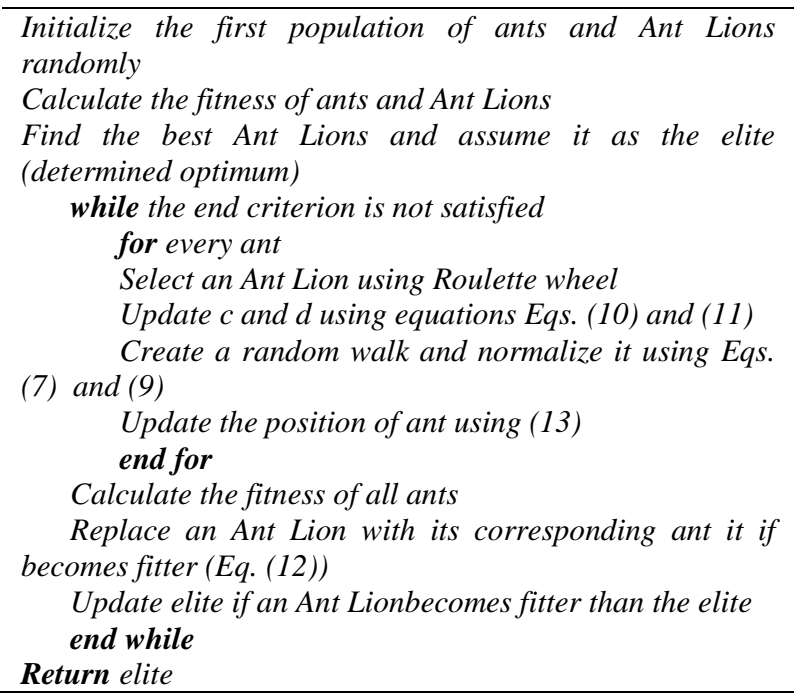

\section{Results and Discussions}

1) IEEE 33-bus test system

This is a medium scale, $12.66 \mathrm{kV}$, radial distribution system with 33 buses. The line, load and tie line data of this test system are taken from [2]. It consists of five tie lines and 32 sectionalize switches. The normally open tie switches are 33-37, and the normally closed sectionalize switches are 1-32.

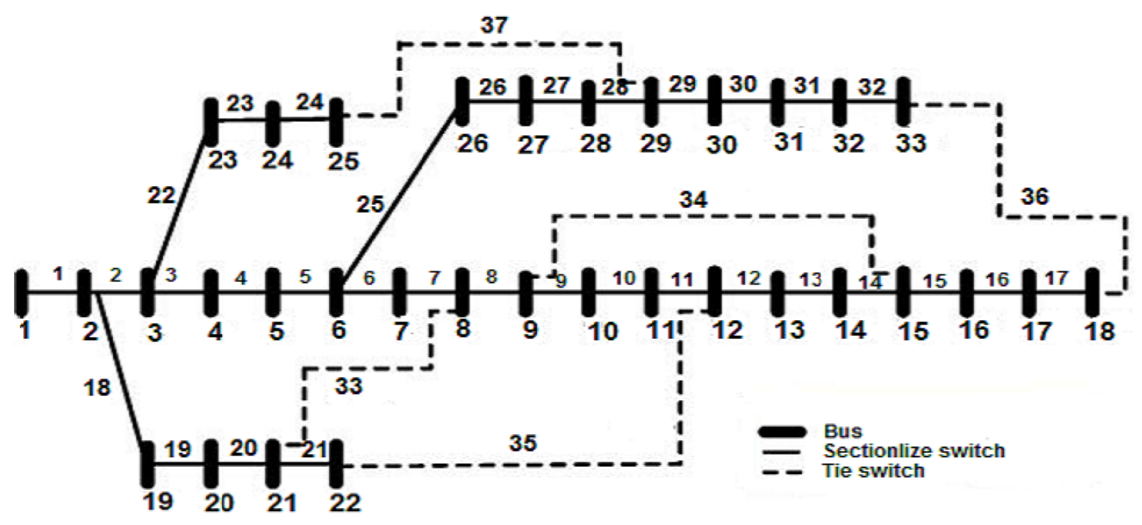

Fig. 1: IEEE 33-bus Distribution System 
The single line diagram of 33-bus system is shown in Fig 1. The dotted lines represent the tie lines. The total real and reactive power loads of the system are 3.72 MW and 2.3 MVAr, respectively. The total real power loss for the base case calculated from power flow is $211.0983 \mathrm{~kW}$. The minimum voltage magnitude of the system is 0.9097 occurs at bus no. 18.

In order to illustrate the convergence ofAL algorithm, the convergence characteristics ofAL algorithm for the best solution is shown in Fig 2. AL algorithm shows a steady and rapid convergence with global searching ability in solving the reconfiguration problem.

The results obtained from theoptimizationshow that thebestconfiguration is when sectionalizing lines\#1, 7, 9, 14and32are open and thentie lines \#3337are close. After thereconfiguration, active power loss issignificantlyreduced $31.3 \%$ as compared to the base case Table 1 . AlsofromthistableandFig 3it isseen that the minimumbusvoltageandsystemvoltage profilearesignificantlyimproved.

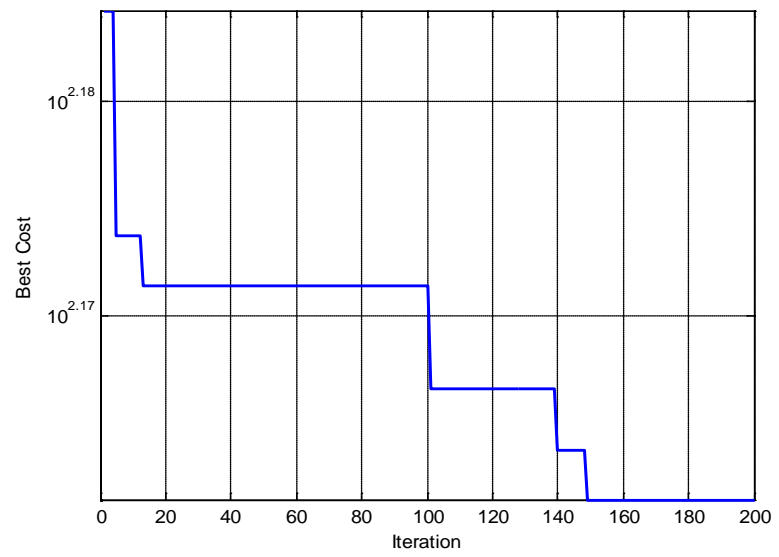

Fig. 2: Convergence Characteristics of AL Algorithm for 33-bus System
Table 1: Simulation results for 33-bus system before and after reconfiguration

\begin{tabular}{|l|c|c|}
\hline & Before & After \\
\hline Line out & -- & $714932 \quad 1$ \\
\hline Tie line & -- & 3334353637 \\
\hline P $_{\text {Loss }}(\mathrm{KW})$ & 211.0983 & 145.0452 \\
\hline Max Voltage (pu) & 1.0000 & 1.0000 \\
\hline Min Voltage (pu) & 0.9097 & 0.9355 \\
\hline
\end{tabular}

\section{2) IEEE 69-bus test system}

This is a medium scale, $12.66 \mathrm{kV}$, radial distribution system with 69 buses. The line, load and tie line data of this test system are taken from [2]. It consists of five tie lines and 69 sectionalize switches. The normally open tie switches are 69-73, and the normally closed sectionalize switches are 1-68. The single line diagram of 69-bus system is shown in Fig 4. The dotted lines represent the tie lines. The total real and reactive power loads of the system are 3.802 MW and 2.6946 MVAr, respectively. The total real power loss for the base case calculated from power flow is $225 \mathrm{~kW}$. The minimum voltage magnitude of the system is 0.9092 occurs at bus no. 54 .

In order to illustrate the convergence of AL algorithm, the convergence characteristics of AL algorithm for the best solution is shown in Fig 5. AL algorithm shows a steady and rapid convergence with global searching ability in solving the reconfiguration problem.

The results obtained from theoptimizationshow that thebestconfiguration is when sectionalizing lines\#12, 17, 33, 45and50are open and thentie lines \#71-73are close. After thereconfiguration, active power loss issignificantlyreduced $55.6 \%$ as compared to the base case, Table 2 . AlsofromthistableandFig 6it isseen that the minimumbusvoltageandsystemvoltage profilearesignificantlyimproved.

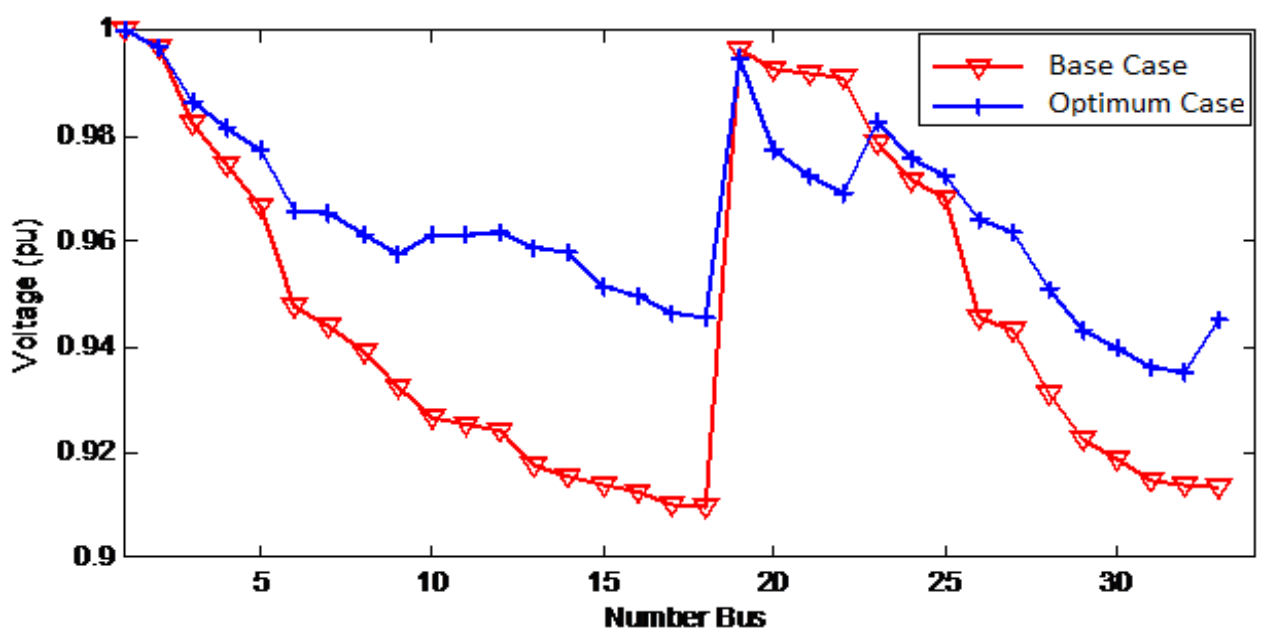

Fig. 3: Comparison of Voltage Magnitude of 33-bus System 


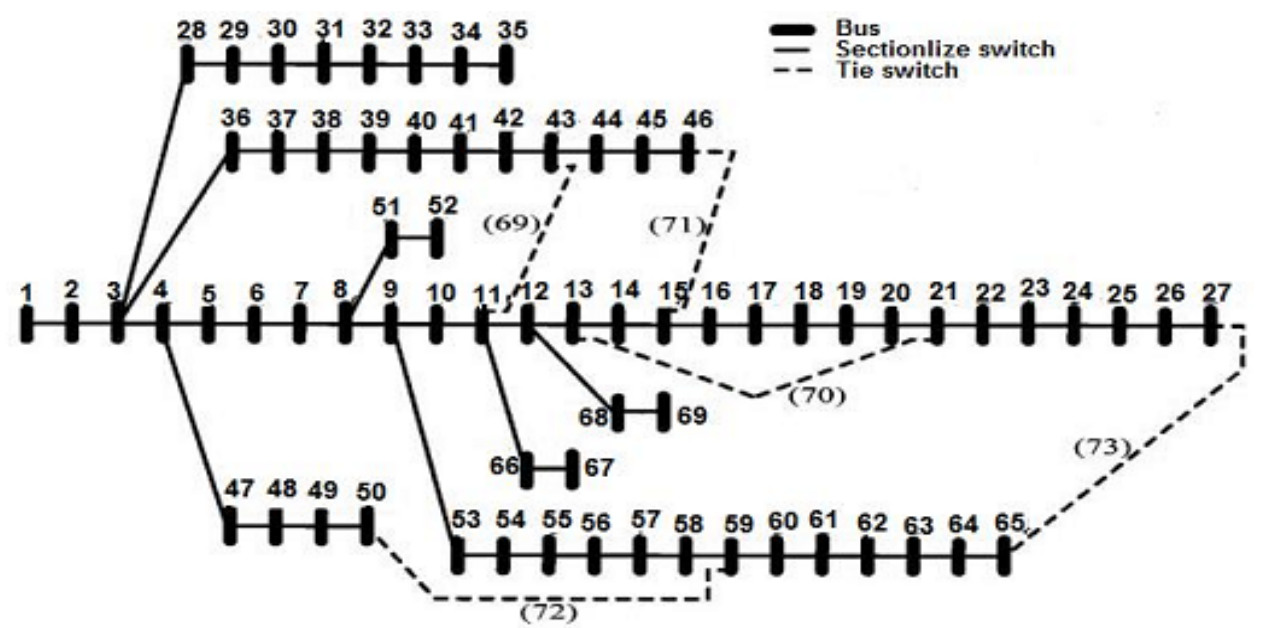

Fig. 4: IEEE 69-bus Distribution System

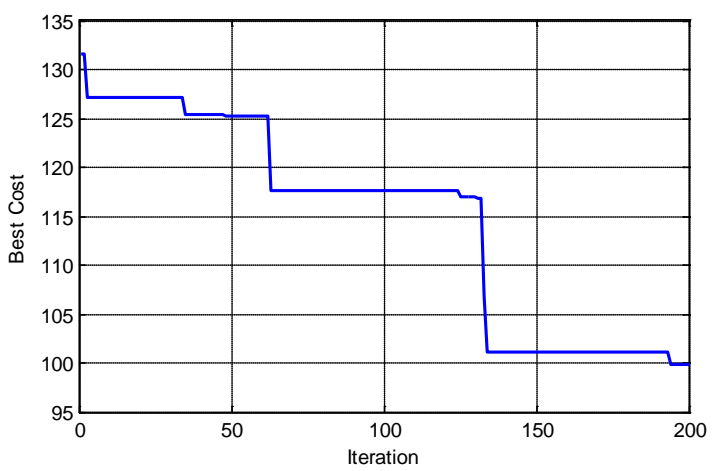

Fig. 5: Convergence Characteristics of AL Algorithm for 69-bus System

Table 2: Simulation results for 69-bus system before and after reconfiguration

\begin{tabular}{|l|c|c|}
\hline & Before & After \\
\hline Line out & -- & 1217334550 \\
\hline Tie line & -- & 717273 \\
\hline $\mathrm{P}_{\text {Loss }}(\mathrm{KW})$ & 225 & 99.8179 \\
\hline Max Voltage (pu) & 1.0000 & 1.0000 \\
\hline Min Voltage (pu) & 0.9092 & 0.9428 \\
\hline
\end{tabular}

\section{Conclusion}

In this paper, meta-heuristic algorithms are used as a new and effective way to solve the reconfiguration problem. The given Optimization algorithm is Ant
Lion algorithm, which is a good way to solve complex optimization problems, such as the reconfiguration. Also, the given algorithm does not need heavy arithmetical calculations. Reconfiguration is a combinational and complex optimization problem and the possible to directly use mathematical optimization methods is difficult. Therefore, in this study, a new methodology based on Ant Lion algorithm is used for reconfiguration because of its high speed and efficiency in complex networks.

Detection and elimination of inappropriate configurations during operation are as advantages of the proposed method that they would increase the run speed. In the proposed method, minimizing the power loss and voltage drop as objective functions and maintaining the radial system structure, bus voltage and line power limitations were considered as reconfiguration problem constraints. The results of simulations performed on 33 \& 69-busIEEEstandard distribution systems represent the high precision and speed of the given algorithm. Evaluation of results shows the high quality of the solutions provided by the Ant Lion algorithm on discrete problems and gives the fact that this optimization method is an appropriate way to solve problems related to distribution networks.

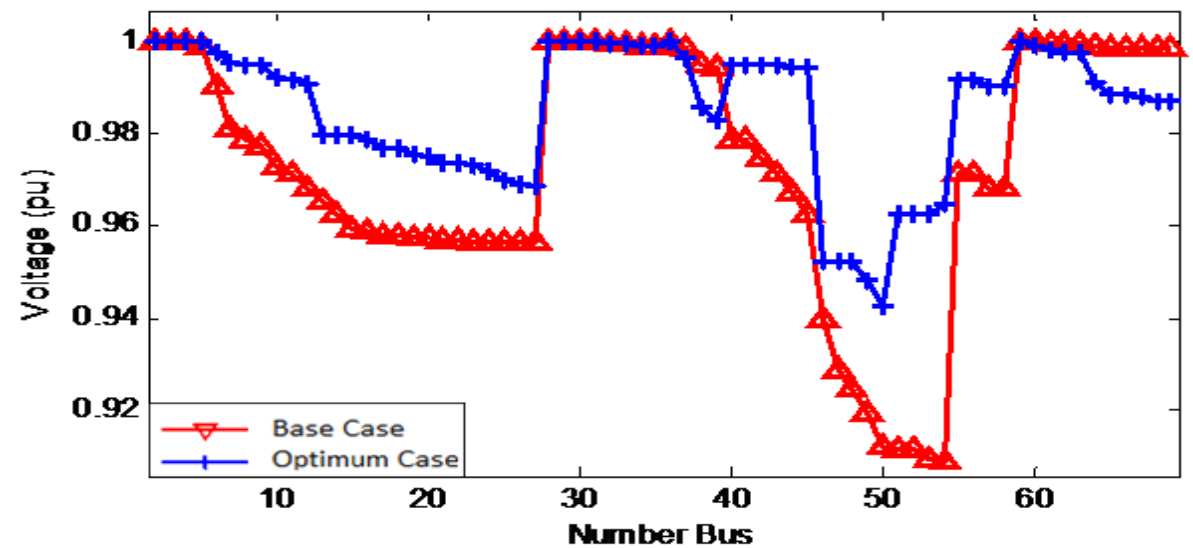

Fig. 6: Comparison of Voltage Magnitude of 69-bus System 


\section{Nomenclature}

$a_{i} \quad$ minimum of random walk of $i$-th variable

Ant $t_{i}^{t} \quad$ position of $i$-th ant at $t$-th iteration

Antlion $_{j}^{t}$ position of selected $j$-th Ant Lionatt-th iteration

$b_{i} \quad$ maximum of random walk in $i$-th variable

$c^{t} \quad$ minimum of all variables at $t$-th iteration

$c_{i}^{t} \quad$ minimum of $i$-th variable at $t$-th iteration

cumsum cumulative sum

$d^{t} \quad$ a vector including the maximum of all

variables at $t$-th iteration

$d_{i}^{t} \quad$ maximum of $i$-th variable at $t$-th iteration

I a ratio

$n \quad$ maximum number of iteration

$Q_{\text {imax }}$ maximum allowable reactive power for capacitor $i$

$Q_{\text {imin }} \quad$ minimum allowable reactive power for capacitor $i$

rand a random number generated with uniform distribution in the interval of $[0,1]$

random walk around the Ant Lion

$R_{A}^{t} \quad$ selected by the roulette wheel at $t$-th iteration

$R_{E}^{t} \quad$ random walk around the elite at $t$-th iteration

$\mathrm{S}_{\mathrm{li} \text { max }} \quad$ maximum active power transmitted from line $i$

step of random walk (iteration in this $t \quad$ study)

$V_{\text {imax }} \quad$ maximum allowable power for bus $i$

$V_{\text {imin }} \quad$ minimum allowable power for bus $i$

$w_{1}, w_{2} \quad$ Constant coefficients

\section{References}

[1] Merlin, A., Back, G. (1975), Search for minimum-loss operational spanning tree configuration for an urban power distribution system,Proc. PSCC’05, Cambridge, pp. 1-18.

[2] Baran, M.E., WuF.F., (1989), Network reconfiguration in distribution systems for loss reduction and load balancing”, IEEETPower Deliver, vol4, no 2, pp. 1401-1407.
[3] Chiou, J., Chang, F., Su, C., (2005) Variable scaling hybrid differential evolution for solving network reconfiguration of distribution systems, IEEE T Power Syst,vol 20, no 2, pp. 668-674.

[4] Sathish-Kumar, K., Jayabarathi, T., (2012) Power system reconfiguration and loss minimization for a distribution systems using bacterial foraging optimization algorithm, Int $\mathrm{J}$ Elec PowerEnergSyst, vol36, no 1, pp. 13-17.

[5] Kashem, M.A., Jlasmon, G.B., Mohamed, A., Moghavvemi, M., (1998) Artificial neural network approach to network reconfiguration for loss minimization in distribution networks, Int J Elec Power EnergSyst, vol 20, no 4, pp. 247-258.

[6] Niknam, T., Azadfarsani, E., Jabbari, M., (2012) A new hybrid evolutionary algorithm based on new fuzzy adaptive PSO and NM algorithms for distribution feeder reconfiguration,Energ Convers Manage, vol54, no 1, pp. 7-16.

[7] Wu, Y., Lee, Ch., Liu, L., Tsai, Sh., (2010) Study of reconfiguration for the distribution system with distributed generators, IEEETPower Deliver, vol25, no 3, pp. 16781685.

[8] Gupta, N., Swarnkar, A., Niazi, K.R., Bansal, R.G., (2012) Multi-objective reconfiguration of distribution systems using adoptive genetic algorithm in fuzzy framework”, IET GenerTransm Dis, vol4, no 12, pp. 1288-1298.

[9] Mendoza, J.E., Lopez, M.E., Coello, C.A.C., Lopez, E.A., (2009) MicrogeneticMultiObgective Reconfiguration Algorithm Considering Power Losses and Reliability Indices for Medium Voltage Distribution Network,IET GenerTransm Dis, vol 3, no 9, pp. 825-840.

[10] Moshtagh, J., Ghasemi, S., (2013) Optimal distribution system reconfiguration using nondominated sorting genetic algorithm (NSGA II), JOperAutom Power Eng, vol1, no 1, pp. 12-21.

[11] MirjaliliS.A.(2015), The Ant Lion optimizer", Adv Eng Softw, vol83, pp. 80-98. 